\title{
Analysis of Eustachian Tube Dysfunction by Dynamic Slow Motion Video Endoscopy and Eustachian Tube Dysfunction Questionnaire in Chronic Otitis Media
}

\author{
Won Gue Han ${ }^{1} \cdot$ Jun Yoo ${ }^{1}$ Yoon Chan Rah ${ }^{1} \cdot$ Jiwon Chang $^{2} \cdot$ Gi Jung $\mathrm{Im}^{1} \cdot$ Jae-Jun Song ${ }^{1}$ Sung Won Chae ${ }^{1}$ \\ Hak Hyun Jung ${ }^{1} \cdot$ June Choi $^{1}$ \\ ${ }^{1}$ Department of Otorhinolaryngology-Head and Neck Surgery, Korea University College of Medicine, Seoul; \\ ${ }^{2}$ Department of Otorhinolaryngology-Head and Neck Surgery, Hallym University College of Medicine, Seoul, Korea
}

Objectives. Eustachian tube dysfunction has been associated with most cases of middle-ear disease. We aimed to assess the effectiveness of dynamic slow motion video endoscopy (DSVE) as a test of eustachian tube dysfunction. Furthermore, we assessed the correlation of the test with the Valsalva maneuver, the seven-item Eustachian Tube Dysfunction Questionnaire (ETDQ-7), and intraoperative findings of the eustachian tube.

Methods. We retrospectively reviewed medical records from April to September 2014 to identify patients who were diagnosed with chronic otitis media (COM) at Korea University Ansan Hospital. They all underwent surgery because of COM without cholesteatoma and were assessed via the DSVE and ETDQ-7 to determine eustachian tube function.

Results. We reviewed $46 \mathrm{COM}$ patients and examined 46 ears with COM and 46 ears on the contralateral side to COM that were thought to be normal. The mean DSVE grade in COM ears was $1.57 \pm 0.96$, while the mean DSVE grade in contralateral ears was $1.15 \pm 0.94$. The difference in DSVE between COM ears and normal ears was statistically significant $(P=0.006)$. In the ETDQ-7, a higher score was related to intraoperative obstruction of the eustachian tube $(P=0.012)$.

Conclusion. DSVE and ETDQ-7 can provide information regarding preoperative status of eustachian tube dysfunction by measuring dynamic structural changes of the eustachian tube in combination with other diagnostic tests.

Keywords. Eustachian Tube; Endoscopy; Otitis Media; Seven-Item Eustachian Tube Dysfunction Questionnaire; Dynamic Slow Motion Video Endoscopy

\section{INTRODUCTION}

The eustachian tube has several functions associated with the middle ear. It promotes ventilation to control air pressure in the

\footnotetext{
- Received December 15, 2016

Revised May 11, 2017

Accepted May 16, 2017

- Corresponding author: June Choi

Department of Otorhinolaryngology-Head and Neck Surgery, Korea

University Ansan Hospital, Korea University College of Medicine,

123 Jeokgeum-ro, Danwon-gu, Ansan 15355, Korea

Tel: +82-31-412-5170, Fax: +82-31-412-5174

E-mail: mednlaw@korea.ac.kr
}

middle ear, drains secretions within the middle ear into the nasopharynx, and protects the middle ear from nasopharyngeal sound pressure and secretions [1]. A decrease in eustachian tube function can affect homeostasis of the middle ear. For instance, in one study, $71.7 \%$ of patients with chronic suppurative otitis media had eustachian tube dysfunction, while eustachian tube dysfunction was observed in $34.9 \%$ of the control group [2]. In many studies, it has been suggested that eustachian tube dysfunction is associated with most cases of middle-ear disease $[3,4]$.

Examining eustachian tube function is one of the most important steps in evaluating the pathogenesis and prognosis of patients with chronic suppurative otitis media. In other words,

Copyright @ 2017 by Korean Society of Otorhinolaryngology-Head and Neck Surgery.

This is an open-access article distributed under the terms of the Creative Commons Attribution Non-Commercial License (http://creativecommons.org/licenses/by-nc/4.0)

which permits unrestricted non-commercial use, distribution, and reproduction in any medium, provided the original work is properly cited. 
when eustachian tube function is preserved, the prognoses for surgeries such as mastoidectomy are more likely to be good [5,6].

Traditionally, there are many tests that evaluate eustachian tube function. In individuals with an intact tympanic membrane, the eustachian tube swallow test assesses changes in applied pressure in the middle ear while swallowing. Tympanometry is performed repeatedly while the examiner adds positive and/or negative pressure to the external auditory canal and asks the patient to swallow [7]. Another test is to perform the Valsalva maneuver, which increases nasopharyngeal pressure and evaluates whether the eustachian tube opens or not [8].

Recently, Poe et al. [9] analyzed eustachian tube dysfunction by video endoscopy in 17 normal individuals and 17 individuals with eustachian tube dysfunction. They demonstrated that slowmotion endoscopic video analysis was a useful new technique for the study of the eustachian tube physiology. Furthermore, many authors have suggested that slow motion video endoscopy is a potentially useful tool for evaluating the pathophysiology of eustachian tube dysfunction [10].

Eustachian tube function can also be evaluated using subjective indicators. The seven-item Eustachian Tube Dysfunction Questionnaire (ETDQ-7) was developed for the assessment of symptoms related to obstructive dysfunction of the eustachian tube and treatment outcomes of middle ear diseases [11].

In the present study, we assessed the effectiveness of dynamic slow motion video endoscopy (DSVE) as a test of eustachian tube dysfunction. Furthermore, we evaluated the strength of correlations of DSVE results with those obtained using the Valsalva maneuver, the ETDQ-7, and intraoperative findings of the eustachian tube.

\section{MATERIALS AND METHODS}

We retrospectively reviewed medical records from April to September 2014 to identify patients who were diagnosed with chronic otitis media $(\mathrm{COM})$ without cholesteatoma at Korea University Ansan Hospital. All patients underwent surgery for COM and were preoperatively evaluated with the DSVE and ETDQ-7 to assess eustachian tube function. Furthermore, medical histo-

\section{H I G H L I G H T S}

- Eustachian tube dysfunction is one of the most important pathogenic factors of chronic otitis media.

- The grade measured by dynamic slow motion video endoscopy was higher in the chronic otitis media ear than in the contralateral ear.

- Higher scores from seven-item Eustachian Tube Dysfunction Questionnaire were related to intraoperative eustachian tube obstruction.
Table 1. Clinical features of subjects

\begin{tabular}{lcc}
\hline Parameter & No. (\%) & Mean \pm SD (yr) \\
\hline Subject sex & & \\
Male & $22(47.8)$ & $45.0 \pm 14.8$ \\
Female & $24(52.2)$ & $51.2 \pm 15.2$ \\
Medical history & & \\
AR & $8(17.4)$ & - \\
GERD & $7(15.2)$ & - \\
Side with COM & & - \\
Right & $18(39.1)$ & - \\
Left & $28(60.9)$ & \\
\hline
\end{tabular}

SD, standard deviation; AR, allergic rhinitis; GERD, gastro-esophageal reflux; COM, chronic otitis media.

ries associated with eustachian tube function such as allergic rhinitis (AR) and gastro-esophageal reflux (GERD) were recorded. This study was reviewed and approved by Korea University Ansan Hospital Institutional Review Board (No. AS16045).

\section{Patients}

A total of 53 patients were enrolled. Among these patients, 7 were excluded due to bilateral COM, and therefore we ultimately included 46 patients in the sample. We did not exclude patients with previous histories of surgery for $\mathrm{COM}$ because previous surgical history was not a confounding factor for assessing the current function of the eustachian tube. The patients included 22 males and 24 females who ranged in age from 17 to 76 years (mean, $48.2 \pm 15.1$ years). The detailed clinical features of patients are shown in Table 1.

\section{Operation procedure}

All patients were examined with preoperative temporal bone computed tomography (CT) for precise evaluation of COM status and were confirmed to have no specific lesions on the contralateral side. All patients underwent unilateral COM operations. The surgical procedures used for $\mathrm{COM}$ were canal up mastoidectomy, canal down mastoidectomy, and radical mastoidectomy with or without tympanoplasty. All surgeries were performed by 1 senior otolaryngologist (JC) in the hospital. The structural evaluation of the eustachian tube orifice during surgery was classified in surgical records as patent or obstructive.

\section{Dynamic slow motion video endoscopy}

For this test, topical epinephrine-soaked gauze (1:10,000 diluted epinephrine, $1 \mathrm{mg} / \mathrm{mL}$; Bosmin, Jeil Pharmaceutical Co. Ltd., Seoul, Korea) was packed in both nasal cavities for 3 minutes. We used a rigid Hopkins rod endoscope (Karl Storz, Tuttlingen, Germany), which had a 30-degree angle and was $4 \mathrm{~mm}$ in diameter. The images and video clips were recorded with an endoscopic camera system (Olympus CLV-S40, Olympus, Seoul, Korea). After the patient was anesthetized, we examined the nasopharyngeal area with an endoscope. The endoscope was intro- 
Table 2. Eustachian tube dysfunction grade according to dynamic slow motion video endoscopy [12]

\begin{tabular}{ll}
\hline Grade & Normal ET with no mucosal edema or congestion. \\
\hline 0 & Medial cartilaginous lamina and lateral wall motions are normal. \\
& Tubal lumen opens well on swallowing. \\
& Edema and congestion of mucosa limited to pharyngeal orifice of ET. \\
& Normal lateral wall motion and tubal lumen opens with swallowing. \\
& Reduced lateral wall motion secondary to edema, congestion involving lumen (2A) and abnormal tubal muscle contraction (2B). \\
3 & Tubal lumen opens partly with swallowing. \\
Patulous (P) & $\begin{array}{l}\text { Pubal lumen fails to open with swallowing secondary to gross edema (3A) and abnormal tubal muscle contraction (3B). } \\
\text { extending toward the isthmus, with medial and lateral cartilaginous lamina remaining separate even at rest. }\end{array}$ \\
\hline
\end{tabular}

ET, eustachian tube.
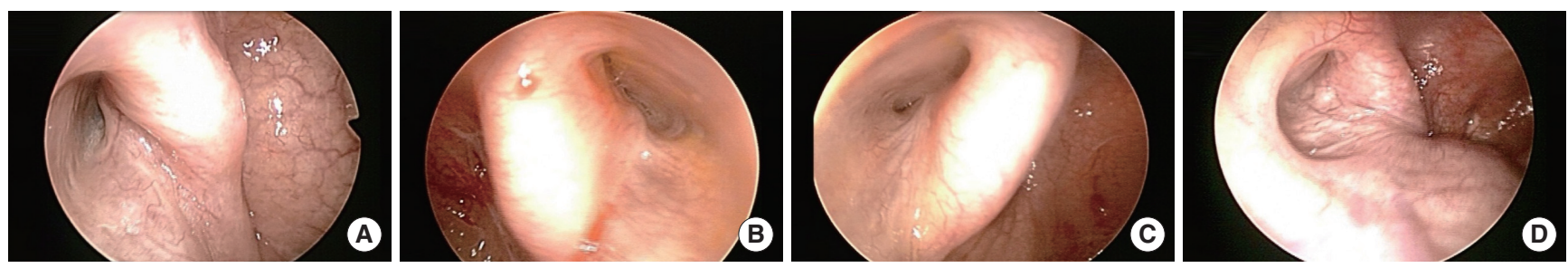

Fig. 1. Endoscopic findings of eustachian tube dysfunction during dynamic slow motion video endoscopy. (A) Tubal lumen opens well during swallowing (grade 0). (B) Edema and congestion of orifice mucosa of eustachian tube (grade 1). (C) Tubal lumen opens partially during swallowing. Edema and congestion involving lumen (grade 2). (D) Tubal lumen fails to open during swallowing (grade 3).

duced along the nasal floor until it faced the nasopharyngeal orifice of the eustachian tube. The patient was asked to swallow saliva and comfortably breathe nasally for 10 seconds. This process was repeated 3 times and the opening or closing of the eustachian tube was recorded and the grade of eustachian tube function was scored. The video recording of nasal endoscopy in slow motion ( 0.2 to 0.4 speed setting) was interpreted and the grade of eustachian tube dysfunction was confirmed by 1 senior otolaryngologist. The grades of eustachian tube dysfunction were scored as shown in Table 2 and Fig. 1 [12].

\section{Seven-item Eustachian Tube Dysfunction Questionnaire}

The ETDQ-7 was administered to all patients before surgery. The ETDQ-7 consists of 7 questions answered on a 7-point scale, with a response of 1 indicating no problem and 7 indicating a severe problem. Total scores range from 7 to 49 [11]. We translated the ETDQ-7 from English to Korean. We assessed the correlation between the original and the translated English version by 2 otologists (JC, KWK). No divergences between the original and translated version were found. The Korean version of the ETDQ-7 was used in this study.

\section{Valsalva maneuver}

The Valsalva maneuver is performed by forceful expiration while keeping the mouth and nose closed until the ears "pop." This technique is useful for equalization of middle ear air pressure. We asked patients to perform the Valsalva maneuver after eval- uating the eustachian tube with DSVE. The patient was asked to blow against air pressure for a few seconds while closing the mouth and holding the nose. After blowing, we assessed changes in the ear fullness or the ear "pop" sensation. If patients felt a decrease in ear fullness or a more ventilated sense in one ear after the Valsalva maneuver, we classified the function of the eustachian tube in that ear as normal. If they felt no change in symptoms, we considered the eustachian tube to be obstructed.

\section{Statistical analyses}

We analyzed the effectiveness of DSVE and the Valsalva maneuver using the Mann-Whitney $U$-test, and compared the reliability of DSVE, the Valsalva maneuver, and ETDQ-7 with intraoperative eustachian tube findings. We also analyzed the severity of eustachian tube dysfunction as evaluated by DSVE and ETDQ-7 according to the existence of AR or GERD using the Mann-Whitney U-test, chi-square test, and Fisher exact test. Furthermore, we examined the correlation between DSVE and ETDQ-7 using Spearman's rho. All statistical analyses were conducted using IBM SPSS ver. 20 (IBM Co., Armonk, NY, USA), and $P<0.05$ was considered statistically significant.

\section{RESULTS}

We reviewed $46 \mathrm{COM}$ patients and examined 46 ears with COM and 46 ears contralateral to COM that were thought to be nor- 
mal. The mean value of DSVE in COM ears was $1.57 \pm 0.96$, while the mean value of DSVE in contralateral ears was $1.15 \pm$ 0.94. The difference in DSVE between COM ears and normal ears was statistically significant $(P=0.037)$. In addition, we affirmed that the grade of DSVE was typically higher in COM ears than in the contralateral ear. We identified $29 \mathrm{COM}$ ears with signs indicating eustachian tube obstruction during the Valsalva maneuver compared to 25 contralateral ears $(P=0.397)$ (Table 3$)$.

Then, we divided the patients into patent $(\mathrm{n}=20)$ vs. obstructive $(n=26)$ groups according to eustachian tube status during surgery, and we examined the compatibility of DSVE, the Valsalva maneuver, and ETDQ-7 score. Higher ETDQ-7 scores were related to intraoperative eustachian tube obstruction $(P=0.010)$. However, the other two factors were not significantly related to intraoperative eustachian tube findings (Table 4).

We also analyzed correlations between DSVE and ETDQ-7 score. Spearman's rho was 0.190 and was not significant $(P=0.206)$. In addition, we analyzed the degree of eustachian tube dysfunction with DSVE and ETDQ-7 according to the existence of AR or GERD. Although patients with AR had higher DSVE and ETDQ-7 values than patients without AR, these differences were not statistically significant. There was no consistent trend relating DSVE and ETDQ-7 in GERD patients. There

Table 3. DSVE grade and the Valsalva maneuver in COM and contralateral ears

\begin{tabular}{lrrc}
\hline Variable & $\begin{array}{c}\text { COM } \\
\text { ear }\end{array}$ & $\begin{array}{c}\text { Contralateral } \\
\text { ear }\end{array}$ & $P$-value \\
\hline DSVE grade & $6(13.0)$ & $12(26.1)$ & \\
0 & $17(37.0)$ & $20(43.5)$ & \\
1 & $14(30.4)$ & $9(19.6)$ & \\
2 & $9(19.6)$ & $5(10.9)$ & \\
3 & $1.57 \pm 0.96$ & $1.15 \pm 0.94$ & - \\
Mean value of DSVE grade & & & $0.397^{\text {b) }}$ \\
Valsalva maneuver & & & \\
\hline The signs of obstructive ET & $29(63.0)$ & $25(54.3)$ & \\
The signs of patent ET & $17(37.0)$ & $21(45.7)$ & \\
\hline
\end{tabular}

Values are presented as number (\%) or mean \pm standard deviation.

COM, chronic otitis media; DSVE, dynamic slow motion video endoscopy;

ET, eustachian tube.

a) Mann-Whitney U-test. ${ }^{\text {b) }}$ Chi-square. $P<0.05$, statistically significant. were no correlations between AR or GERD and intraoperative eustachian tube obstruction (Table 5).

\section{DISCUSSION}

The eustachian tube plays key roles in the pathogenesis of various middle-ear diseases including acute or chronic suppurative otitis media, otitis media with effusion, and middle-ear atelectasis [13]. Preoperative and postoperative tubal function is important for good surgical outcomes in patients with COM [13]. A number of tests to evaluate eustachian tube function have been developed, and we focused on the DSVE, the Valsalva maneuver, and ETDQ-7 in this study.

We demonstrated that the mean DSVE value was higher in $\mathrm{COM}$ ears than in contralateral ears. In addition, we confirmed that the grade of DSVE in the COM ear was typically higher than that of the contralateral ear. Therefore, DSVE may be a good diagnostic tool for assessing the dynamic structure of the eustachian tube. Augustine et al. [14] examined 100 clinically normal middle ears to evaluate eustachian tube function and found that tympanometric findings were correlated with DSVE findings via the eustachian tube swallow test. They concluded that although DSVE appeared to over-diagnose eustachian tube dysfunction, it was useful as a simple, easy procedure for assessing eustachian tube function when combined with other eustachian tube function tests [14]. In another study, 200 ears were tested to examine and compare the efficacy of DSVE in evaluat-

Table 4. Compatibility of DSVE, the Valsalva maneuver, and ETDQ-7 with intraoperative eustachian tube findings

\begin{tabular}{lccc}
\hline Vatiable & $\begin{array}{c}\text { ET obstruction } \\
(\mathrm{n}=26)\end{array}$ & $\begin{array}{c}\text { ET patent } \\
(\mathrm{n}=20)\end{array}$ & $P$-value \\
\hline DSVE grade & $1.65 \pm 0.98$ & $1.45 \pm 0.94$ & $0.479^{\mathrm{a})}$ \\
ETDQ-7 score & $18.58 \pm 8.28$ & $12.70 \pm 3.88$ & $0.010^{\mathrm{a})}$ \\
Valsalva maneuver & & & $0.765^{\mathrm{b})}$ \\
Signs of obstructive ET & $17(65.4)$ & $12(60.0)$ & \\
\hline
\end{tabular}

Values are presented as number (\%) or mean \pm standard deviation. $E T$, eustachian tube; DSVE, dynamic slow motion video endoscopy; ETDQ-7, the seven-item Eustachian Tube Dysfunction Questionnaire. ${ }^{\text {a)} M a n n-W h i t n e y ~ U-t e s t . ~}{ }^{\text {b)}}$ Chi-square. $P<0.05$, statistically significant.

Table 5. Degree of eustachian tube dysfunction evaluated by DSVE and ETDQ-7 according to the presence of AR or GERD

\begin{tabular}{|c|c|c|c|c|c|c|}
\hline \multirow{2}{*}{ Variable } & \multicolumn{3}{|c|}{$\mathrm{AR}$} & \multicolumn{3}{|c|}{ GERD } \\
\hline & Presence $(n=8)$ & Not $(n=38)$ & $P$-value & Presence $(n=7)$ & Not $(n=39)$ & $P$-value \\
\hline DSVE grade & $1.62 \pm 1.12$ & $1.55 \pm 0.92$ & $0.787^{\mathrm{a})}$ & $1.71 \pm 1.11$ & $1.54 \pm 0.94$ & $0.675^{a)}$ \\
\hline ETDQ-7 score & $17.75 \pm 8.65$ & $15.66 \pm 7.05$ & $0.467^{\mathrm{a})}$ & $13.86 \pm 4.22$ & $16.41 \pm 7.68$ & $0.675^{\text {a) }}$ \\
\hline ET obstruction finding during surgery & $3(37.5)$ & $17(44.7)$ & $1.000^{\mathrm{b})}$ & $2(28.6)$ & $18(46.2)$ & $1.000^{c)}$ \\
\hline
\end{tabular}

Values are presented as number (\%) or mean \pm standard deviation.

AR, allergic rhinitis; GERD, gastro-esophageal reflux; DSVE, dynamic slow motion video endoscopy; ETDQ-7, The seven-item Eustachian Tube Dysfunction Questionnaire; ET, eustachian tube.

a) Mann-Whitney U-test. ${ }^{\text {b)}}$ Chi-square. ${ }^{\mathrm{c}}$ Fisher exact test. $P<0.05$, statistically significant. 
ing eustachian tube dysfunction to that of tympanometry. The combination of DSVE and tympanometry was found to be a useful testing modality for evaluating eustachian tube status in patients with chronic middle ear diseases [15].

In the present study, we also used the Valsalva maneuver to evaluate eustachian tube dysfunction, but did not find any significant correlation between signs indicating eustachian tube obstruction during the Valsalva maneuver and intraoperative eustachian tube findings. Our results are different from those of a previous study that reported no significant differences in the success rates of middle ear pressure equalization techniques [16]. That study reported that the Valsalva and Toynbee maneuvers showed $51.7 \%$ effectiveness for evaluating eustachian tube function while the Ear Popper showed 43.3\% effectiveness [16]. The diagnostic sensitivity was $95 \%$ and specificity was $83 \%$ when all tests (Valsalva maneuver, eustachian tube opening pressure, dilatory efficiency, and percentage of positive pressure equilibrated) were combined [17].

We confirmed that patients with intraoperative eustachian tube obstruction had higher ETDQ-7 scores than patients with patent eustachian tubes. We detected a correlation between eustachian tube dysfunction and ETDQ-7. These findings are consistent with those of Van Roeyen et al. [18], who reviewed 39 patients with eustachian tube obstruction and found that the ETDQ-7 was a useful scale for assessing eustachian tube dysfunction. There is some controversy concerning the relationship between AR and eustachian tube dysfunction. In the current study, we did not find any correlations between AR and degree of eustachian tube dysfunction. Our results agree with the results of earlier studies, which reported that abnormalities in eustachian tube function are not significantly different between AR patients and non-AR patients [19]. However, other authors have reported that AR patients have higher risk of eustachian tube dysfunction than those without AR [20].

Although the number of patients included in the present study was small, our findings did not reveal any correlations between GERD and eustachian tube dysfunction, in contrast to previous studies suggesting that GERD may be related to eustachian tube dysfunction $[19,20]$. Although DSVE and ETDQ-7 have some limitations for our purposes, many authors have determined that they may be good diagnostic tests when performed alongside other tests of eustachian tube dysfunction [11-14]. We suggest that the DSVE is useful as a simple diagnostic tool for assessing the dynamic structure of the eustachian tube before surgery for the treatment of COM. In addition, the ETDQ-7 may be a good method for estimating the opening of the intraoperative eustachian tube before surgery.

Limitations of our study include the lack of specific information about tympanometry when we performed the Valsalva maneuver in patients. Additionally, we detected no correlation between DSVE and ETDQ-7, which may be due to our small sample size or recall bias on the ETDQ-7, which asks patients to describe symptoms occurring within the last month. Moreover, we did not compare results from the DSVE or ETDQ-7 to those obtained when performing the swallow test with tympanometry. In the future, we will conduct studies which include larger sample sizes and more diagnostic tools for comparing DSVE and ETDQ-7.

In conclusion, we confirmed that the dynamic structure of the eustachian tube is more occluded in ears affected by COM than in the contralateral (normal) ear when analyzed with DSVE. In addition, ETDQ-7 score was correlated with the existence of intraoperative eustachian tube obstruction. Therefore, DSVE and ETDQ-7, in combination with other diagnostic tests, can provide useful information regarding the preoperative status of eustachian tube dysfunction related to dynamic structural changes in the eustachian tube.

\section{CONFLICT OF INTEREST}

No potential conflict of interest relevant to this article was reported.

\section{ACKNOWLEDGMENTS}

We are grateful to Kang Woo Kim for giving efforts for assessing the correlation between the original and the translated English version.

\section{REFERENCES}

1. Bluestone CD, Doyle WJ. Anatomy and physiology of eustachian tube and middle ear related to otitis media. J Allergy Clin Immunol. 1988 May;81(5 Pt 2):997-1003.

2. Yuceturk AV, Unlu HH, Okumus M, Yildiz T, Filiz U. The evaluation of eustachian tube function in patients with chronic otitis media. Clin Otolaryngol Allied Sci. 1997 Oct;22(5):449-52.

3. Sente M. Importance of the eustachian tube in middle ear function. Med Pregl. 1995;48(11-12):405-6.

4. Seibert JW, Danner CJ. Eustachian tube function and the middle ear. Otolaryngol Clin North Am. 2006 Dec;39(6):1221-35.

5. Iwano T, Kinoshita T, Hamada E, Doi T, Ushiro K, Kumazawa T. Otitis media with effusion and eustachian tube dysfunction in adults and children. Acta Otolaryngol Suppl. 1993;500:66-9.

6. Bluestone CD. Current management of chronic suppurative otitis media in infants and children. Pediatr Infect Dis J. 1988 Nov;7(11 Suppl):S137-40.

7. Williams PS. A tympanometric pressure swallow test for assessment of eustachian tube function. Ann Otol Rhinol Laryngol. 1975 MayJun;84(3 Pt 1):339-43.

8. Bluestone CD, Cantekin EI. Current clinical methods, indications and interpretation of eustachian tube function tests. Ann Otol Rhinol Laryngol. 1981 Nov-Dec;90(6 Pt 1):552-62.

9. Poe DS, Pyykko I, Valtonen H, Silvola J. Analysis of eustachian tube function by video endoscopy. Am J Otol. 2000 Sep;21(5):602-7.

10. Poe DS, Abou-Halawa A, Abdel-Razek O. Analysis of the dysfunctional eustachian tube by video endoscopy. Otol Neurotol. 2001 Sep; 
22(5):590-5.

11. McCoul ED, Anand VK, Christos PJ. Validating the clinical assessment of eustachian tube dysfunction: the Eustachian Tube Dysfunction Questionnaire (ETDQ-7). Laryngoscope. 2012 May;122(5): 1137-41.

12. Mathew GA, Kuruvilla G, Job A. Dynamic slow motion video endoscopy in eustachian tube assessment. Am J Otolaryngol. 2007 MarApr;28(2):91-7.

13. Tos M. Importance of eustachian tube function in middle ear surgery. Ear Nose Throat J. 1998 Sep;77(9):744-7.

14. Augustine AM, Varghese L, Michael RC, Albert RR, Job A. The efficacy of dynamic slow motion video endoscopy as a test of eustachian tube function. J Laryngol Otol. 2013 Jul;127(7):650-5.

15. Padhya C, Sharma Y, Mishra G. To assess the efficacy of modality slow motion dynamic video endoscopy of eustachian tube in chronic middle ear pathologies. Indian J Otolaryngol Head Neck Surg. 2015 Mar;67(1):81-7.
16. Hidir Y, Ulus S, Karahatay S, Satar B. A comparative study on efficiency of middle ear pressure equalization techniques in healthy volunteers. Auris Nasus Larynx. 2011Aug;38(4):450-5.

17. Doyle WJ, Swarts JD, Banks J, Casselbrant ML, Mandel EM, Alper CM. Sensitivity and specificity of eustachian tube function tests in adults. JAMA Otolaryngol Head Neck Surg. 2013 Jul;139(7):71927.

18. Van Roeyen S, Van de Heyning P, Van Rompaey V. Value and discriminative power of the seven-item Eustachian Tube Dysfunction Questionnaire. Laryngoscope. 2015 Nov;125(11):2553-6.

19. Sente M, Sente R, Puleva K, Milekic NK. Allergic rhinitis as a possible etiologic cause eustachian tube dysfunction. Med Pregl. 2001 Mar-Apr;54(3-4):166-71.

20. Lazo-Saenz JG, Galvan-Aguilera AA, Martinez-Ordaz VA, VelascoRodriguez VM, Nieves-Renteria A, Rincon-Castaneda C. Eustachian tube dysfunction in allergic rhinitis. Otolaryngol Head Neck Surg. 2005 Apr;132(4):626-9. 